\title{
Fast Sampling Control of Singularly Perturbed Systems with Actuator Saturation and $L_{2}$ Disturbance
}

\author{
Yanzi Miao, Lei Ma, Xiaoping Ma, and Linna Zhou \\ School of Information and Electrical Engineering, China University of Mining and Technology, Xuzhou 221116, China \\ Correspondence should be addressed to Xiaoping Ma; xiaopingmacumt@126.com
}

Received 27 April 2015; Accepted 3 August 2015

Academic Editor: Wanquan Liu

Copyright (C) 2015 Yanzi Miao et al. This is an open access article distributed under the Creative Commons Attribution License, which permits unrestricted use, distribution, and reproduction in any medium, provided the original work is properly cited.

\begin{abstract}
We will consider the problem of fast sampling control for singularly perturbed systems subject to actuator saturation and $L_{2}$ disturbance. A sufficient condition for the existence of a state feedback controller is proposed. Under this controller, the boundedness of the trajectories in the presence of $L_{2}$ disturbances is guaranteed for any singular perturbation parameter less than or equal to a predefined upper bound. To improve the capacity of disturbance tolerance and disturbance rejection, two convex optimization problems are formulated. Finally, a numerical example is presented to demonstrate the effectiveness of the main results of this paper.
\end{abstract}

\section{Introduction}

Many practical physical systems consist of subsystems operating on different time scales. Applying the normal control methods to these systems usually lead to ill-conditioned numerical problems. To overcome the numerical problem, singular perturbation theory was introduced to the field of control system and widely used in practice [1]. In this framework, a multiple time-scale system is modeled by a singularly perturbed system (SPS) with a small positive parameter such that the degree of separation between fast and slow modes can be determined. For example, in a power system, the flux linkages of the rotor windings are fast variables while the emf behind transient reactance, the generator's rotor angle in radians, and the actual rotor speed are the slow ones. Thus the power system can be modeled as a SPS, where the torque of the winding represents the perturbation parameter $\varepsilon$ [2].

Since most of the modern control systems are implemented by computer, sampling control of SPSs has been widely investigated. There are three sampling modes for SPSs: multirate, slow, and fast sampling mode. Under multirate sampling mode, the slow and fast states are measured at different sampling rate. In [3], a multirate sampling model predictive control method is proposed for large-scale nonlinear uncertain systems. In [4], a multirate sampling composite controller is designed. Slow sampling control is usually under the assumption that the fast subsystem is stable $[5,6]$. Slow sampling discrete-time SPS is considered in $[7,8]$. If the fast subsystem is not stable, fast sampling control is necessary $[9,10]$. Fast sampling control of SPSs with disturbance is considered in [10-12]. In [11], $H_{\infty}$ controller design method together with a stability bound optimization method is proposed, and a less conservative method is proposed in [12]. But the above achievements do not take into account the actuator saturation, which is common in practice. It is known that actuator saturation may force the systems to work in the open-loop and thus destroy stability of control systems [13]. Thus many research efforts have been devoted to analysis and design of control systems with actuator saturation [14-19]. Recently, SPSs with actuator saturation are considered. Besides basin of attraction, stability bound is also an important stability index for SPSs with actuator saturation. In [20-24], state feedback controller is designed and the basin of attraction is estimated. The obtained results guarantee the existence of the stability bound but can not present an estimate of the bound. Many results have been proposed for estimating or enlarging the stability bound of the SPSs without actuator saturation [25-32]. In [33], continuoustime SPS with actuator saturation is considered and a state feedback controller is designed to achieve a desired stability 
bound while the basin of attraction is optimized. To the best knowledge of the authors, the fast sampling control problem of the SPSs with actuator saturation and disturbance has not been considered.

This paper focuses on fast sampling control of SPSs subject to actuator saturation and $L_{2}$ disturbance. First, a state feedback controller design method is proposed such that the trajectories of the closed-loop SPSs starting from a bounded set remain bounded for any allowable singular perturbation parameter and $L_{2}$ disturbance. Then, a method to enlarge the capacity of disturbance tolerance is proposed in terms of linear matrix inequalities (LMIs). Furthermore, a convex optimization problem is formulated to optimize the disturbance rejection. Finally, an example is given to show the effectiveness of the proposed results.

The rest of this paper is organized as follows: Section 2 provides the problems under consideration. Section 3 gives the main results of this paper. In Section 4, an example is presented to demonstrate the proposed approaches. Section 5 makes a conclusion of the paper.

Notations. The superscript $T$ stands for matrix transpose. For a matrix $M$, the notation $M^{-T}$ denotes the transpose of the inverse matrix of $M$ and $M_{(i)}$ denotes $i$ th row of $M$. Let $Q \in R^{n \times n}$ be a positive definite matrix. An ellipsoid $\Omega(Q, \rho)$ is defined as $\Omega(Q, \rho) \triangleq\left\{\eta \in R^{n} \mid \eta^{T} Q \eta \leq \rho\right\}$.

\section{Problem Formulation}

The fast sampling model of SPSs with actuator saturation and $L_{2}$ disturbance is described by the following compact form:

$$
\begin{aligned}
x(k+1) & =A_{\varepsilon} x(k)+B_{\varepsilon} \text { sat }(u(k))+E_{\varepsilon} w(k), \\
z(k) & =C x(k),
\end{aligned}
$$

where

$$
\begin{aligned}
x(k) & =\left[\begin{array}{l}
x_{1}(k) \\
x_{2}(k)
\end{array}\right] \in R^{n}, \\
A_{\varepsilon} & =\left[\begin{array}{cc}
I+\varepsilon A_{11} & \varepsilon A_{12} \\
A_{21} & A_{22}
\end{array}\right], \\
B_{\varepsilon} & =\left[\begin{array}{c}
\varepsilon B_{1} \\
B_{2}
\end{array}\right], \\
E_{\varepsilon} & =\left[\begin{array}{c}
\varepsilon E_{1} \\
E_{2}
\end{array}\right], \\
C & =\left[\begin{array}{ll}
C_{1} & C_{2}
\end{array}\right] .
\end{aligned}
$$

$\varepsilon$ represents the singular perturbation parameter and is assumed to be available for controller design in this paper. $x_{1} \in R^{n_{1}}$ and $x_{2} \in R^{n_{2}}$ are the state variables, $u(k)$ is the control input, $z(k)$ is the output of the system, $A_{11}, A_{12}, A_{21}$, $A_{22}, B_{1}, B_{2}$ and $E_{1}, E_{2}$ are constant matrices of appropriate dimensions and $\operatorname{sat}(\cdot)$ is a componentwise saturation map
$R^{m} \mapsto R^{m}$ defined by $\operatorname{sat}\left(u_{j}\right)=\operatorname{sign}\left(u_{j}\right) \min \left\{1,\left|u_{j}\right|\right\}, j=$ $1,2, \ldots, m$, and $w(k)$ is the $L_{2}$ disturbance which belongs to

$$
W_{\alpha}^{2}:=\left\{w: R_{+} \longrightarrow R^{q}: \sum_{k=0}^{+\infty} w^{T}(k) w(k) \leq \alpha\right\},
$$

where $\alpha$ is a positive number.

Under the state feedback controller

$$
u(k)=K_{\varepsilon} x(k),
$$

the closed-loop system can be described by

$$
\begin{aligned}
x(k+1) & =A_{\varepsilon} x(k)+B_{\varepsilon} \operatorname{sat}\left(K_{\varepsilon} x(k)\right)+E_{\varepsilon} w(k), \\
z(k) & =C x(k) .
\end{aligned}
$$

The problems under consideration are as follows.

Problem 1. Given $\varepsilon_{0}>0$ and $\alpha^{*}>0$, design a state feedback controller (4), such that, for any $\varepsilon \in\left(0, \varepsilon_{0}\right]$ and $w \in W_{\alpha^{*}}^{2}$, all the trajectories of the closed-loop system starting from inside in $\Omega\left(P^{-1}(\varepsilon), 1\right)$ will remain inside of $\Omega\left(P^{-1}(\varepsilon), 1+\eta \alpha^{*}\right)$ with $\eta>0$ and $P(\varepsilon)>0$ to be determined.

Problem 2. Given $\varepsilon_{0}>0$, design a state feedback controller (4) to maximize $\alpha^{*}$, such that for any $\varepsilon \in\left(0, \varepsilon_{0}\right]$ and $w \in W_{\alpha^{*}}^{2}$ all the trajectories of the closed-loop system starting from the origin are bounded.

Problem 3. Given $\varepsilon_{0}>0$ and $\alpha^{*}>0$, design a state feedback controller (4) to minimize $\gamma>0$, such that, for any $\varepsilon \in$ $\left(0, \varepsilon_{0}\right]$ and $w \in W_{\alpha^{*}}^{2}$, the restricted $L_{2}$ gain from output to disturbance of the closed-loop system with zero initial condition is less than $\gamma$ under the control of (4).

Remark 4. The upper bound $\varepsilon_{0}$ characterizes the robustness of the system performance with respect to $\varepsilon$. The disturbance tolerance bound $\alpha^{*}$ describes the largest disturbance that can be tolerated. The disturbance rejection $\gamma$ means the largest ratio between the $L_{2}$ norms of the output and the disturbance.

There are two common tools to handle the saturation nonlinearities, one is the sector bound approach [34], and the other is the convex hull approach $[13,18]$. The latter is adopted in this paper since it has been shown to be less conservative than the former [18]. The related preliminaries are recalled in the following.

For a given matrix $K \in R^{m \times n}$, denote the $i$ th row of matrix $K$ as $k_{i}$ and define

$$
\mathscr{L}(K)=\left\{\eta \in R^{n}:\left|k_{i} \eta\right| \leq 1, i \in[1, m]\right\} .
$$

Let $\mathscr{D}$ be the set of $m \times m$ diagonal matrices whose diagonal elements are either 1 or 0 . There are $2^{m}$ elements in $\mathscr{D}$. Suppose these elements of $\mathscr{D}$ are labeled as $D_{i}, i \in\left[1,2^{m}\right]$. Denote $D_{i}^{-}=I-D_{i}$. Clearly, $D_{i}^{-} \in \mathscr{D}$ if $D_{i} \in \mathscr{D}$.

Lemma 5 (see [13]). Let $K, H \in R^{m \times n}$. Then, for any $x \in$ $\mathscr{L}(H)$, it holds that

$$
\text { sat }(K x) \in \operatorname{co}\left\{D_{i} K x+D_{i}^{-} H x, i \in\left[1,2^{m}\right]\right\},
$$

where co stands for the convex hull. 


\section{Main Results}

3.1. Controller Design. This subsection will present a solution to Problem 1.

Theorem 6. Given $\varepsilon_{0}>0$ and $\alpha^{*}>0$, if there exist symmetric matrices $P_{11} \in R^{n_{1} * n_{1}}, P_{22} \in R^{n_{2} * n_{2}}$ and matrices $P_{12} \in R^{n_{1} * n_{2}}$, $Z_{1} \in R^{m * n_{1}}, Z_{2} \in R^{m * n_{2}}, Y_{1} \in R^{m * n_{1}}, Y_{2} \in R^{m * n_{2}}$, as well as a positive scalar $\eta$ satisfying

$$
\begin{aligned}
& {\left[\begin{array}{cccc}
P_{22} & \phi_{1}^{T} & \phi_{2}^{T} & 0 \\
\star & -\phi_{3}-\phi_{3}^{T} & P_{12}-\phi_{4}^{T} & E_{1} \\
\star & \star & P_{22} & E_{2} \\
\star & \star & \star & \eta I
\end{array}\right]>0, \quad i \in\left[1,2^{m}\right]} \\
& {\left[\begin{array}{ccccc}
\varepsilon_{0} P_{11} & \varepsilon_{0} P_{12} & \varepsilon_{0} \phi_{3}^{T} & \varepsilon_{0} \phi_{4}^{T} & 0 \\
\star & P_{22} & \varepsilon_{0} P_{12}^{T} A_{11}^{T}+\phi_{1}^{T} & \varepsilon_{0} P_{12}^{T} A_{21}^{T}+\phi_{2}^{T} & 0 \\
\star & \star & -\phi_{3}-\phi_{3}^{T} & P_{12}-\phi_{4}^{T} & E_{1} \\
\star & \star & \star & P_{22} & E_{2} \\
\star & \star & \star & \star & \eta I
\end{array}\right]} \\
& >0, \quad i \in\left[1,2^{m}\right], \\
& {\left[\begin{array}{cc}
P_{22} & Y_{2(r)}^{T} \\
Y_{2(r)} & \frac{1}{1+\eta \alpha^{*}}
\end{array}\right]>0, \quad r=1,2, \ldots, m,} \\
& {\left[\begin{array}{ccc}
\varepsilon_{0} P_{11} & \varepsilon_{0} P_{12} & \varepsilon_{0} Y_{1(r)}^{T} \\
\varepsilon_{0} P_{12}^{T} & P_{22} & Y_{2(r)}^{T} \\
\varepsilon_{0} Y_{1(r)}^{T} & Y_{2(r)} & \frac{1}{1+\eta \alpha^{*}}
\end{array}\right]>0, \quad r=1,2, \ldots, m,} \\
& \phi_{1}=A_{12} P_{22}+B_{1} D_{i} Z_{2}+B_{1} D_{i}^{-} Y_{2} \text {, } \\
& \phi_{2}=A_{22} P_{22}+B_{2} D_{i} Z_{2}+B_{2} D_{i}^{-} Y_{2} \text {, } \\
& \phi_{3}=A_{11} P_{11}+A_{12} P_{12}^{T}+B_{1} D_{i} Z_{1}+B_{1} D_{i}^{-} Y_{1} \text {, } \\
& \phi_{4}=A_{21} P_{11}+B_{2} D_{i} Z_{1}+B_{2} D_{i}^{-} Y_{1}+A_{22} P_{12}^{T} \text {, } \\
& i \in\left[1,2^{m}\right],
\end{aligned}
$$

then, for any $\varepsilon \in\left(0, \varepsilon_{0}\right]$ and $w \in W_{\alpha^{*}}^{2}$, all of the trajectories of the closed-loop system (5) starting within $\Omega\left(P^{-1}(\varepsilon), 1\right)$ will remain inside of $\Omega\left(P^{-1}(\varepsilon), 1+\eta \alpha^{*}\right)$ with $P(\varepsilon)=\left[\begin{array}{cc}\varepsilon P_{11} & \varepsilon P_{12} \\ \varepsilon P_{12}^{T} & P_{22}\end{array}\right]>0$.

And the state feedback controller gain matrix is given by

$$
K_{\varepsilon}=\left[\begin{array}{ll}
Z_{1} & Z_{2}
\end{array}\right]\left[\begin{array}{cc}
P_{11} & \varepsilon P_{12} \\
P_{12}^{T} & P_{22}
\end{array}\right]^{-1} .
$$

Proof. From (10) and (11), it follows that, for any $\varepsilon \in\left(0, \varepsilon_{0}\right]$,

$$
\left[\begin{array}{ccc}
\varepsilon P_{11} & \varepsilon P_{12} & \varepsilon Y_{1(r)}^{T} \\
\varepsilon P_{12}^{T} & P_{22} & Y_{2(r)}^{T} \\
\varepsilon Y_{1(r)}^{T} & Y_{2(r)} & \frac{1}{1+\eta \alpha^{*}}
\end{array}\right]>0, \quad r=1,2, \ldots, m
$$
lent to

Let $Z_{\varepsilon}=\left[\begin{array}{ll}\varepsilon Z_{1} & Z_{2}\end{array}\right], Y_{\varepsilon}=\left[\begin{array}{ll}\varepsilon Y_{1} & Y_{2}\end{array}\right]$. Then (14) is equiva-

$$
\left[\begin{array}{cc}
P(\varepsilon) & Y_{\varepsilon(r)}^{T} \\
Y_{\varepsilon(r)} & \frac{1}{1+\eta \alpha^{*}}
\end{array}\right]>0, \quad r=1,2, \ldots, m
$$

which implies that

$$
\left[\begin{array}{cc}
P^{-1}(\varepsilon) & H_{\varepsilon(r)}^{T} \\
H_{\varepsilon(r)} & \frac{1}{1+\eta \alpha^{*}}
\end{array}\right]>0, \quad r=1,2, \ldots, m,
$$

where $H_{\varepsilon}=Y_{\varepsilon} P^{-1}(\varepsilon)$.

By Schur complement, it follows from (16) that

$$
P^{-1}(\varepsilon)>H_{\varepsilon(r)}^{T}\left(1+\eta \alpha^{*}\right) H_{\varepsilon(r)}, \quad r=1,2, \ldots, m,
$$

which implies that $\Omega\left(P^{-1}(\varepsilon), 1+\eta \alpha^{*}\right) \subseteq \mathscr{L}\left(H_{\varepsilon}\right), \forall \varepsilon \in\left(0, \varepsilon_{0}\right]$. Then by Lemma 5, we have

$$
\begin{aligned}
& A_{\varepsilon} x(k)+B_{\varepsilon} \operatorname{sat}\left(K_{\varepsilon} x(k)\right) \\
& \quad \in \operatorname{co}\left\{A_{\varepsilon} x(k)+B_{\varepsilon}\left(D_{i} K_{\varepsilon}+D_{i}^{-} H_{\varepsilon}\right) x(k)\right\}, \\
& \quad \forall \varepsilon \in\left(0, \varepsilon_{0}\right], i \in\left[1,2^{m}\right] .
\end{aligned}
$$

From (8) and (9), it follows that

$$
\left[\begin{array}{ccccc}
\varepsilon P_{11} & \varepsilon P_{12} & \varepsilon \phi_{3}^{T} & \varepsilon \phi_{4}^{T} & 0 \\
\star & P_{22} & \varepsilon P_{12}^{T} A_{11}^{T}+\phi_{1}^{T} & \varepsilon P_{12}^{T} A_{21}^{T}+\phi_{2}^{T} & 0 \\
\star & \star & -\phi_{3}-\phi_{3}^{T} & P_{12}-\phi_{4}^{T} & E_{1} \\
\star & \star & \star & P_{22} & E_{2} \\
\star & \star & \star & \star & \eta I
\end{array}\right]>0,
$$

$$
\forall \varepsilon \in\left(0, \varepsilon_{0}\right], i \in\left[1,2^{m}\right] \text {. }
$$

Before and after multiplying (19) by

$$
\left[\begin{array}{lllll}
I & 0 & 0 & 0 & 0 \\
0 & I & 0 & 0 & 0 \\
0 & 0 & \varepsilon I & 0 & 0 \\
0 & 0 & 0 & I & 0 \\
0 & 0 & 0 & 0 & I
\end{array}\right]
$$


and its transpose, respectively, we have

$$
\left[\begin{array}{ccccc}
\varepsilon P_{11} & \varepsilon P_{12} & \varepsilon^{2} \phi_{3}^{T} & \varepsilon \phi_{4}^{T} & 0 \\
\star & P_{22} & \varepsilon^{2} P_{12}^{T} A_{11}^{T}+\varepsilon \phi_{1}^{T} & \varepsilon P_{12}^{T} A_{21}^{T}+\phi_{2}^{T} & 0 \\
\star & \star & \varepsilon^{2}\left(-\phi_{3}-\phi_{3}^{T}\right) & \varepsilon P_{12}-\varepsilon \phi_{4}^{T} & \varepsilon E_{1} \\
\star & \star & \star & P_{22} & E_{2} \\
\star & \star & \star & \star & \eta I
\end{array}\right]
$$$$
>0, \quad \forall \varepsilon \in\left(0, \varepsilon_{0}\right], \quad i \in\left[1,2^{m}\right] \text {. }
$$

Before and after multiplying (21) by the matrix

$$
\left[\begin{array}{lllll}
I & 0 & 0 & 0 & 0 \\
0 & I & 0 & 0 & 0 \\
I & 0 & I & 0 & 0 \\
0 & 0 & 0 & I & 0 \\
0 & 0 & 0 & 0 & I
\end{array}\right]
$$

and its transpose, respectively, we have

$$
\left[\begin{array}{ccccc}
\varepsilon P_{11} & \varepsilon P_{12} & \varepsilon P_{11}+\varepsilon^{2} \phi_{3}^{T} & \varepsilon \phi_{4}^{T} & 0 \\
\star & P_{22} & \varepsilon P_{12}^{T}+\varepsilon^{2} P_{12}^{T} A_{11}^{T}+\varepsilon \phi_{1}^{T} & \varepsilon P_{12}^{T} A_{21}^{T}+\phi_{2}^{T} & 0 \\
\star & \star & \varepsilon P_{11} & \varepsilon P_{12} & \varepsilon E_{1} \\
\star & \star & \star & P_{22} & E_{2} \\
\star & \star & \star & \star & \eta I
\end{array}\right]
$$

$>0, \quad \forall \varepsilon \in\left(0, \varepsilon_{0}\right], i \in\left[1,2^{m}\right]$.

Taking into account the definitions of $A_{\varepsilon}, B_{\varepsilon}, E_{\varepsilon}$, and $P(\varepsilon)$, we can rewrite $(23)$ as

$$
\left[\begin{array}{ccc}
P(\varepsilon) & \left(A_{\varepsilon} P(\varepsilon)+B_{\varepsilon} D_{i} Z_{\varepsilon}+B_{\varepsilon} D_{i}^{-} Y_{\varepsilon}\right)^{T} & 0 \\
\star & P(\varepsilon) & E_{\varepsilon} \\
\star & \star & \eta I
\end{array}\right]>0,
$$

$$
\forall \varepsilon \in\left(0, \varepsilon_{0}\right], i \in\left[1,2^{m}\right] \text {. }
$$

Define

$$
K_{\varepsilon}=Z_{\varepsilon} P^{-1}(\varepsilon)
$$

Before and after multiplying (24) by $\operatorname{diag}\left(P^{-1}(\varepsilon), P^{-1}(\varepsilon)\right.$, I), we have

$$
\left[\begin{array}{ccc}
P^{-1}(\varepsilon) & \left(A_{\varepsilon}+B_{\varepsilon} D_{i} K_{\varepsilon}+B_{\varepsilon} D_{i}^{-} H_{\varepsilon}\right)^{T} P^{-1}(\varepsilon) & 0 \\
\star & P^{-1}(\varepsilon) & P^{-1}(\varepsilon) E_{\varepsilon} \\
\star & \star & \eta I
\end{array}\right]
$$

$>0, \quad \forall \varepsilon \in\left(0, \varepsilon_{0}\right], i \in\left[1,2^{m}\right]$.

By Schur complement, inequality (26) is equivalent to

$$
\left[\begin{array}{cc}
\Xi_{1} & \Xi_{2} \\
\Xi_{2}^{T} & \Xi_{3}-\eta I
\end{array}\right]<0,
$$

where

$$
\begin{aligned}
\Xi_{1} & =\left(A_{\varepsilon}+B_{\varepsilon} D_{i} K_{\varepsilon}+B_{\varepsilon} D_{i}^{-} H_{\varepsilon}\right)^{T} P^{-1}(\varepsilon) \\
& \cdot\left(A_{\varepsilon}+B_{\varepsilon} D_{i} K_{\varepsilon}+B_{\varepsilon} D_{i}^{-} H_{\varepsilon}\right)-P^{-1}(\varepsilon), \\
\Xi_{2} & =\left(A_{\varepsilon}+B_{\varepsilon} D_{i} K_{\varepsilon}+B_{\varepsilon} D_{i}^{-} H_{\varepsilon}\right)^{T} P^{-1}(\varepsilon) E_{\varepsilon}, \\
\Xi_{3} & =E_{\varepsilon}^{T} P^{-1}(\varepsilon) E_{\varepsilon},
\end{aligned}
$$

$$
\forall \varepsilon \in\left(0, \varepsilon_{0}\right], i \in\left[1,2^{m}\right] \text {. }
$$

Define an $\varepsilon$-dependent Lyapunov function:

$$
V(x)=x^{T} P^{-1}(\varepsilon) x .
$$

Calculating the difference of $V(k)$ along the trajectories of the closed-loop system (5), and using (18), we have

$$
\begin{aligned}
& \Delta V(x)=V(x(k+1))-V(x(k))=\left(A_{\varepsilon} x(k)\right. \\
& \left.+B_{\varepsilon} \operatorname{sat}(u)+E_{\varepsilon} w(k)\right)^{T} P^{-1}(\varepsilon)\left(A_{\varepsilon} x(k)+B_{\varepsilon} \text { sat }(u)\right. \\
& \left.+E_{\varepsilon} w(k)\right)-x^{T}(k) P^{-1}(\varepsilon) x(k) \\
& \leq \max _{i \in\left[1,2^{m}\right]}\left\{\left(A_{\varepsilon} x(k)+B_{\varepsilon}\left(D_{i} K_{\varepsilon}+D_{i}^{-} H_{\varepsilon}\right) x(k)\right.\right. \\
& \left.+E_{\varepsilon} w(k)\right)^{T} P^{-1}(\varepsilon)\left(A_{\varepsilon} x(k)+B_{\varepsilon}\left(D_{i} K_{\varepsilon}+D_{i}^{-} H_{\varepsilon}\right)\right. \\
& \left.\left.\cdot x(k)+E_{\varepsilon} w(k)\right)-x^{T}(k) P^{-1}(\varepsilon) x(k)\right\} \\
& =\max _{i \in\left[1,2^{m}\right]}\left\{x ^ { T } ( k ) \left[\left(A_{\varepsilon}+B_{\varepsilon}\left(D_{i} K_{\varepsilon}+D_{i}^{-1} H_{\varepsilon}\right)\right)^{T}\right.\right. \\
& \left.\cdot P^{-1}(\varepsilon)\left(A_{\varepsilon}+B_{\varepsilon}\left(D_{i} K_{\varepsilon}+D_{i}^{-1} H_{\varepsilon}\right)\right)-P^{-1}(\varepsilon)\right] \\
& \cdot x(k)+x^{T}(k)\left[A_{\varepsilon}+B_{\varepsilon}\left(D_{i} K_{\varepsilon}+D_{i}^{-1} H_{\varepsilon}\right)\right]^{T} \\
& \cdot P^{-1}(\varepsilon) E_{\varepsilon} w(k)+w^{T}(k) E_{\varepsilon}^{T} P^{-1}(\varepsilon)\left[A_{\varepsilon}\right. \\
& \left.+B_{\varepsilon}\left(D_{i} K_{\varepsilon}+D_{i}^{-1} H_{\varepsilon}\right)\right] x(k)+w^{T}(k) E_{\varepsilon}^{T} P^{-1}(\varepsilon) \\
& \left.\cdot E_{\varepsilon} w(k)\right\}, \\
& \forall x(k) \in \Omega\left(P^{-1}(\varepsilon), 1+\eta \alpha^{*}\right), \varepsilon \in\left(0, \varepsilon_{0}\right], w \in W_{\alpha^{*}}^{2}
\end{aligned}
$$

It follows that

$$
\begin{aligned}
& \Delta V(x)-\eta w^{T}(k) w(k)=\xi^{T}(k)\left[\begin{array}{cc}
\Xi_{1} & \Xi_{2} \\
\Xi_{2}^{T} & \Xi_{3}-\eta I
\end{array}\right] \xi(k) \\
& \quad \leq 0
\end{aligned}
$$

$\forall x(k) \in\left(\Omega\left(P^{-1}(\varepsilon), 1+\eta \alpha^{*}\right)\right), \varepsilon \in\left(0, \varepsilon_{0}\right], w \in W_{\alpha^{*}}^{2}$,

with

$$
\xi(k)=\left[\begin{array}{l}
x(k) \\
w(k)
\end{array}\right] .
$$


Then we have

$\Delta V(x) \leq \eta w^{T}(k) w(k)$,

$\forall x(k) \in \Omega\left(P^{-1}(\varepsilon), 1+\eta \alpha^{*}\right), \varepsilon \in\left(0, \varepsilon_{0}\right], w \in W_{\alpha^{*}}^{2}$.

Summing up both sides of (33) from 0 to $m$, we can get

$$
\begin{aligned}
V(x(m+1)) & \leq V(x(0))+\eta \sum_{k=0}^{m} w^{T}(k) w(k) \\
& \leq V(x(0))+\alpha \eta
\end{aligned}
$$

$\forall x(k) \in \Omega\left(P^{-1}(\varepsilon), 1+\eta \alpha^{*}\right), \varepsilon \in\left(0, \varepsilon_{0}\right], w \in W_{\alpha^{*}}^{2}$,

which shows that when $x(0) \in \Omega\left(P^{-1}(\varepsilon), 1\right)$, we can get $V(x(m+1)) \leq 1+\eta \alpha^{*}$, that is, $x(m+1) \in \Omega\left(P^{-1}(\varepsilon), 1+\eta \alpha^{*}\right)$.

And, according to (25), the controller gain matrix is

$$
\begin{aligned}
K_{\varepsilon} & =Z_{\varepsilon} P^{-1}(\varepsilon)=\left[\begin{array}{ll}
\varepsilon Z_{1} & Z_{2}
\end{array}\right]\left[\begin{array}{cc}
\varepsilon P_{11} & \varepsilon P_{12} \\
\varepsilon P_{12}^{T} & P_{22}
\end{array}\right]^{-1} \\
& =\left[\begin{array}{ll}
Z_{1} & Z_{2}
\end{array}\right]\left[\begin{array}{cc}
P_{11} & \varepsilon P_{12} \\
P_{12}^{T} & P_{22}
\end{array}\right]^{-1} .
\end{aligned}
$$

Remark 7. According to (33), when the disturbance $w(k)=$ 0 , it holds that $\Delta V(x)<0, \forall x \neq 0$, which means that the closed-loop system (5) is locally asymptotically stable. In this case, the ellipsoid $\Omega\left(P^{-1}(\varepsilon), 1\right)$ is an estimation of the basin of attraction of the closed-loop system. In addition, when $\varepsilon$ is small enough, an $\varepsilon$-independent gain matrix can be computed by

$$
\begin{aligned}
& K=\lim _{\varepsilon \rightarrow 0^{+}}\left[\begin{array}{ll}
Z_{1} & Z_{2}
\end{array}\right]\left[\begin{array}{cc}
P_{11} & \varepsilon P_{12} \\
P_{12}^{T} & P_{22}
\end{array}\right]^{-1} \\
& =\left[\begin{array}{ll}
Z_{1} & Z_{2}
\end{array}\right]\left[\begin{array}{cc}
P_{11} & 0 \\
P_{12}^{T} & P_{22}
\end{array}\right]^{-1} \\
& =\left[\left(Z_{1}-Z_{2} P_{22}^{-1} P_{12}^{T}\right) P_{11}^{-1} Z_{2} P_{22}^{T}\right] \text {. }
\end{aligned}
$$

3.2. Disturbance Tolerance. The ability of the closed-loop system to tolerate the disturbance is characterized by $\alpha^{*}$. Based on Theorem 6 , we have the following corollary which can be used to maximize $\alpha^{*}$.

Corollary 8. Given $\varepsilon_{0}>0$ and $\alpha>0$, if there exist symmetric matrices $P_{11} \in R^{n_{1} * n_{1}}, P_{22} \in R^{n_{2} * n_{2}}$ and matrices $P_{12} \in R^{n_{1} * n_{2}}$, $Z_{1} \in R^{m * n_{1}}, Z_{2} \in R^{m * n_{2}}, Y_{1} \in R^{m * n_{1}}, Y_{2} \in R^{m * n_{2}}$ satisfying

$$
\left[\begin{array}{cccc}
P_{22} & \phi_{1}^{T} & \phi_{2}^{T} & 0 \\
\star & -\phi_{3}-\phi_{3}^{T} & P_{12}-\phi_{4}^{T} & E_{1} \\
\star & \star & P_{22} & E_{2} \\
\star & \star & \star & I
\end{array}\right]>0, \quad i \in\left[1,2^{m}\right]
$$

$$
\begin{aligned}
& {\left[\begin{array}{ccccc}
\varepsilon_{0} P_{11} & \varepsilon_{0} P_{12} & \varepsilon_{0} \phi_{3}^{T} & \varepsilon_{0} \phi_{4}^{T} & 0 \\
\star & P_{22} & \varepsilon_{0} P_{12}^{T} A_{11}^{T}+\phi_{1}^{T} & \varepsilon_{0} P_{12}^{T} A_{21}^{T}+\phi_{2}^{T} & 0 \\
\star & \star & -\phi_{3}-\phi_{3}^{T} & P_{12}-\phi_{4}^{T} & E_{1} \\
\star & \star & \star & P_{22} & E_{2} \\
\star & \star & \star & \star & I
\end{array}\right]} \\
& >0, \quad i \in\left[1,2^{m}\right], \\
& {\left[\begin{array}{cc}
P_{22} & Y_{2(r)} \\
Y_{2(r)}^{T} & \frac{1}{\alpha}
\end{array}\right]>0 \text {, }} \\
& {\left[\begin{array}{ccc}
\varepsilon_{0} P_{11} & \varepsilon_{0} P_{12} & \varepsilon_{0} Y_{1(r)} \\
\varepsilon_{0} P_{12}^{T} & P_{22} & Y_{2(r)} \\
\varepsilon_{0} Y_{1(r)}^{T} & Y_{2(r)}^{T} & \frac{1}{\alpha}
\end{array}\right]>0,}
\end{aligned}
$$

where $\phi_{1}, \phi_{2}, \phi_{3}, \phi_{4}$ are defined in Theorem 6 , then all the trajectories of the closed-loop system (5) starting from the origin will still remain inside of $\Omega\left(P^{-1}(\varepsilon), \alpha\right)$. And the state feedback controller gain matrix is given by

$$
K_{\varepsilon}=\left[\begin{array}{ll}
Z_{1} & Z_{2}
\end{array}\right]\left[\begin{array}{cc}
P_{11} & \varepsilon P_{12} \\
P_{12}^{T} & P_{22}
\end{array}\right]^{-1} .
$$

Proof. According to (39) and (40), for any $\varepsilon \in\left(0, \varepsilon_{0}\right]$, we have

$$
\left[\begin{array}{ccc}
\varepsilon P_{11} & \varepsilon P_{12} & \varepsilon Y_{1(r)} \\
\varepsilon P_{12}^{T} & P_{22} & Y_{2(r)} \\
\varepsilon Y_{1(r)}^{T} & Y_{2(r)}^{T} & \frac{1}{\alpha}
\end{array}\right]>0
$$

which is equivalent to

$$
\left[\begin{array}{cc}
P^{-1}(\varepsilon) & H_{\varepsilon(r)}^{T} \\
H_{\varepsilon(r)} & \frac{1}{\alpha}
\end{array}\right]>0, \quad r=1,2, \ldots, m .
$$

By Schur complement, it follows from (43) that

$$
P^{-1}(\varepsilon)>H_{\varepsilon(r)}^{T} \alpha H_{\varepsilon(r)}, \quad r=1,2, \ldots, m ;
$$

thus $\Omega\left(P^{-1}(\varepsilon), \alpha\right) \subseteq L\left(H_{\varepsilon}\right), \forall \varepsilon \in\left(0, \varepsilon_{0}\right]$.

Similarly to (34) in the proof for Theorem 6, for the trajectories start from the origin, we have

$$
\begin{aligned}
V(x(m+1)) & \leq V(0)+\sum_{k=0}^{m} w^{T}(k) w(k) \leq V(0)+\alpha \\
& =\alpha .
\end{aligned}
$$

This complete the proof.

From Corollary 8 , the bigger $\alpha$ means the better disturbance tolerance ability. To get the best disturbance tolerance ability we formulate the following optimization problem:

$$
\begin{aligned}
\max _{P_{11}, P_{12}, P_{22}, Z_{1}, Z_{2}, Y_{1}, Y_{2}} & \alpha \\
\text { s.t. } & (37),(38),(39),(40),
\end{aligned}
$$

where $\Omega(S, 1)$ represents the initial condition set. 
Let $\mu=1 / \alpha$. Then inequalities (39) and (40) can be rewritten as

$$
\begin{gathered}
{\left[\begin{array}{cc}
P_{22} & Y_{2(r)}^{T} \\
Y_{2(r)} & \mu
\end{array}\right]>0, \quad r=1,2, \ldots, m,} \\
{\left[\begin{array}{ccc}
\varepsilon_{0} P_{11} & \varepsilon_{0} P_{12} & \varepsilon_{0} Y_{1(r)}^{T} \\
\varepsilon_{0} P_{12}^{T} & P_{22} & Y_{2(r)}^{T} \\
\varepsilon_{0} Y_{1(r)}^{T} & Y_{2(r)} & \mu
\end{array}\right]>0, \quad r=1,2, \ldots, m .}
\end{gathered}
$$
into

Then the optimization problem (46) can be converted $\min _{P_{11}, P_{12}, P_{22}, Z_{1}, Z_{2}, Y_{1}, Y_{2}} \mu$ s.t. (37), (38), (47).

Solving the optimization problem (48) yields the minimal value $\mu^{*}$. Then the largest disturbance that can be tolerated by the closed-loop system at zero initial condition is bounded by $\alpha^{*}=1 / \mu^{*}$. Therefore, Problem 2 can be solved by the optimization problem (48).

3.3. Disturbance Rejection. Problem 3 will be considered in this subsection.

Theorem 9. Given $\varepsilon_{0}>0, \alpha^{*}>0$, and $\gamma>0$, if there exist symmetric matrices $P_{11} \in R^{n_{1} * n_{1}}, P_{22} \in R^{n_{2} * n_{2}}$ and matrices $P_{12} \in R^{n_{1} * n_{2}}, Z_{1} \in R^{m * n_{1}}, Z_{2} \in R^{m * n_{2}}, Y_{1} \in R^{m * n_{1}}, Y_{2} \in R^{m * n_{2}}$ satisfying

$$
\begin{aligned}
& {\left[\begin{array}{ccccc}
P_{22} & \phi_{1}^{T} & \phi_{2}^{T} & 0 & P_{22} C_{2}^{T} \\
\star & -\phi_{3}-\phi_{3}^{T} & P_{12}-\phi_{4}^{T} & E_{1} & -\phi_{5}^{T} \\
\star & \star & P_{22} & E_{2} & 0 \\
\star & \star & \star & \gamma^{2} I & 0 \\
\star & \star & \star & \star & I
\end{array}\right]>0} \\
& {\left[\begin{array}{cccccc}
\varepsilon_{0} P_{11} & \varepsilon_{0} P_{12} & \varepsilon_{0} \phi_{3}^{T} & \varepsilon_{0} \phi_{4}^{T} & 0 & \varepsilon_{0} \phi_{5}^{T} \\
\star & P_{22} & \varepsilon_{0} P_{12}^{T} A_{11}^{T}+\phi_{1}^{T} & \varepsilon_{0} P_{12}^{T} A_{21}^{T}+\phi_{2}^{T} & 0 & \varepsilon_{0} P_{12}^{T} C_{1}^{T}+P_{22} C_{2}^{T} \\
\star & \star & -\phi_{3}-\phi_{3}^{T} & P_{12}-\phi_{4}^{T} & E_{1} & -\phi_{5}^{T} \\
\star & \star & \star & P_{22} & E_{2} & 0 \\
\star & \star & \star & \star & \gamma^{2} I & 0 \\
\star & \star & \star & \star & \star & I
\end{array}\right]>0, \quad i \in\left[1,2^{m}\right],} \\
& {\left[\begin{array}{cc}
P_{22} & Y_{2} \\
Y_{2}^{T} & \frac{1}{\alpha^{*}}
\end{array}\right]>0,} \\
& {\left[\begin{array}{ccc}
\varepsilon_{0} P_{11} & \varepsilon_{0} P_{12} & \varepsilon_{0} Y_{1} \\
\varepsilon_{0} P_{12}^{T} & P_{22} & Y_{2} \\
\varepsilon_{0} Y_{1}^{T} & Y_{2}^{T} & \frac{1}{\alpha^{*}}
\end{array}\right]>0,}
\end{aligned}
$$

where

$$
\begin{aligned}
& \phi_{1}=A_{12} P_{22}+B_{1} D_{i} Z_{2}+B_{1} D_{i}^{-} Y_{2}, \\
& \phi_{2}=A_{22} P_{22}+B_{2} D_{i} Z_{2}+B_{2} D_{i}^{-} Y_{2}, \\
& \phi_{3}=A_{11} P_{11}+A_{12} P_{12}^{T}+B_{1} D_{i} Z_{1}+B_{1} D_{i}^{-} Y_{1}, \\
& \phi_{4}=A_{21} P_{11}+B_{2} D_{i} Z_{1}+B_{2} D_{i}^{-} Y_{1}+A_{22} P_{12}^{T}, \\
& \qquad i \in\left[1,2^{m}\right], \\
& \phi_{5}=C_{1} P_{11}+C_{2} P_{12},
\end{aligned}
$$

then the $L_{2}$ gain from $w$ to $z$ of the closed-loop system (5) with $x(0)=0$ is less than $\gamma$. And the state feedback controller gain matrix is given by

$$
K_{\varepsilon}=\left[\begin{array}{ll}
Z_{1} & Z_{2}
\end{array}\right]\left[\begin{array}{cc}
P_{11} & \varepsilon P_{12} \\
P_{12}^{T} & P_{22}
\end{array}\right]^{-1} .
$$

Proof. Let $P(\varepsilon)=\left[\begin{array}{ll}\varepsilon P_{11} & P_{12} \\ \varepsilon P_{12}^{T} & P_{22}\end{array}\right]>0, Z_{\varepsilon}=\left[\begin{array}{ll}\varepsilon Z_{1} & Z_{2}\end{array}\right], Y_{\varepsilon}=$ $\left[\begin{array}{ll}\varepsilon Y_{1} & Y_{2}\end{array}\right], H_{\varepsilon}=Y_{\varepsilon} P^{-1}(\varepsilon), K_{\varepsilon}=Z_{\varepsilon} P^{-1}(\varepsilon)$. 
Similarly to the proof for Theorem 6, it follows from (49) and (50) that

$$
\left[\begin{array}{cccc}
P(\varepsilon) & \left(A_{\varepsilon} P(\varepsilon)+B_{\varepsilon} D_{i} Z_{\varepsilon}+B_{\varepsilon} D_{i}^{-} Y_{\varepsilon}\right)^{T} & 0 & P(\varepsilon) C^{T} \\
\star & P(\varepsilon) & E_{\varepsilon} & 0 \\
\star & \star & \gamma^{2} I & 0 \\
\star & \star & \star & I
\end{array}\right]
$$

$>0, \quad \forall \varepsilon \in\left(0, \varepsilon_{0}\right], i \in\left[1,2^{m}\right]$.

Applying the Schur complement formula to (55), we have

$$
\left[\begin{array}{cc}
\Xi_{1}+C^{T} C & \Xi_{2} \\
\Xi_{2}^{T} & \Xi_{3}-\gamma^{2} I
\end{array}\right]<0,
$$

where

$$
\begin{aligned}
\Xi_{1} & =\left(A_{\varepsilon}+B_{\varepsilon} D_{i} K_{\varepsilon}+B_{\varepsilon} D_{i}^{-} H_{\varepsilon}\right)^{T} P^{-1}(\varepsilon) \\
& \cdot\left(A_{\varepsilon}+B_{\varepsilon} D_{i} K_{\varepsilon}+B_{\varepsilon} D_{i}^{-} H_{\varepsilon}\right)-P^{-1}(\varepsilon), \\
\Xi_{2} & =\left(A_{\varepsilon}+B_{\varepsilon} D_{i} K_{\varepsilon}+B_{\varepsilon} D_{i}^{-} H_{\varepsilon}\right)^{T} P^{-1}(\varepsilon) E_{\varepsilon}, \\
\Xi_{3} & =E_{\varepsilon}^{T} P^{-1}(\varepsilon) E_{\varepsilon},
\end{aligned}
$$

$$
\forall \varepsilon \in\left(0, \varepsilon_{0}\right], i \in\left[1,2^{m}\right] .
$$

From (51) and (52), for any $\varepsilon \in\left(0, \varepsilon_{0}\right]$, it follows that

$$
\left[\begin{array}{ccc}
\varepsilon P_{11} & \varepsilon P_{12} & \varepsilon Y_{1} \\
\varepsilon P_{12}^{T} & P_{22} & Y_{2} \\
\varepsilon Y_{1}^{T} & Y_{2}^{T} & \frac{1}{\alpha^{*}}
\end{array}\right]>0
$$

From the proof for Theorem 6 , we can get

$$
P^{-1}(\varepsilon)>H_{\varepsilon(r)}^{T} \alpha H_{\varepsilon(r)}, \quad r=1,2, \ldots, m,
$$

which shows that $\Omega\left(P^{-1}(\varepsilon), \alpha^{*}\right) \subseteq \mathscr{L}\left(H_{\varepsilon}\right), \forall \varepsilon \in\left(0, \varepsilon_{0}\right]$.

Define an $\varepsilon$-dependent Lyapunov function

$$
V(x)=x^{T} P^{-1}(\varepsilon) x .
$$

Similarly to proof for Theorem 6 , it follows from (56) that

$$
\begin{array}{r}
\Delta V(x(k))+z^{T}(k) z(k)-\gamma^{2} w^{T}(k) w(k)<0, \\
\forall x \in \Omega\left(P^{-1}(\varepsilon), \alpha^{*}\right) .
\end{array}
$$

Then, summing up left and right of (61), respectively, with $x(0)=0$, yields that

$$
\begin{aligned}
& V(x(m+1))-0 \\
& \quad+\sum_{k=0}^{m}\left(z^{T}(k) z(k)-\gamma^{2} w^{T}(k) w(k)\right)<0,
\end{aligned}
$$

which implies

$$
\sum_{k=0}^{m} z^{T}(k) z(k)<\gamma^{2} \sum_{k=0}^{j} w^{T}(k) w(k) .
$$

Thus the $L_{2}$ gain from $w$ to $z$ of the closed-loop system with $x(0)=0$ is less than $\gamma$. This completes the proof.
By Theorem 9, the minimal $L_{2}$ gain can be obtained by solving the following optimization problem:

$$
\begin{aligned}
\min _{P_{11}, P_{12}, P_{22}, Z_{1}, Z_{2}, Y_{1}, Y_{2}} & \gamma^{2} \\
\text { s.t. } & (49),(50),(51),(52) .
\end{aligned}
$$

Remark 10. As mentioned in Section 1, discretization of a continuous-time SPS can lead to different discrete-time models depending on the sampling rate. Since the structure of fast sampling models is different from that of slow sampling models, it is not easy to generalize the proposed results to slow sampling control of SPSs, as will be considered in our future work.

\section{Examples}

This section will illustrate the proposed results by an example. Consider an inverted pendulum system controlled by DC motor via a gear train. The model, which was first established in [35], is described by

$$
\begin{aligned}
\dot{x}_{1}(t) & =x_{2}(t), \\
\dot{x}_{2}(t) & =\frac{g}{l} \sin x_{1}(t)+\frac{N K_{m}}{m l^{2}} x_{3}(t), \\
L_{a} \dot{x}_{3}(t) & =-K_{b} N x_{2}(t)-R_{a} x_{3}(t)+u(t)+w(t),
\end{aligned}
$$

where $x_{1}(t)=\theta_{p}(t)$ denotes the the angle ( $\left.\mathrm{rad}\right)$ of the pendulum from the vertical upward, $x_{2}(t)=\dot{\theta}_{p}(t), x_{3}(t)=$ $I_{a}(t)$ denotes the current of the motor, $u(t)$ is the control input voltage, $w(t)$ is the disturbance, $K_{m}$ is the motor torque constant, $K_{b}$ is the back emf constant, $N$ is the gear ratio, and $L_{a}$ is the inductance which is usually a small positive constant. The parameters for the plant are as follows: $g=$ $9.8 \mathrm{~m} / \mathrm{s}^{2}, N=10, l=1 \mathrm{~m}, m=1 \mathrm{~kg}, K_{m}=0.1 \mathrm{Nm} / \mathrm{A}, K_{b}=$ $0.1 \mathrm{Vs} / \mathrm{rad}, R_{a}=1 \Omega$, and $L_{a}=0.05 \mathrm{H}$ and the input voltage is required to satisfy $|u| \leq 1$. Note that $L_{a}$ represents the singular perturbation parameter of the system. Substituting the parameters into (65) and linearizing the equations, we have

$$
\begin{aligned}
\dot{x}_{1}(t) & =x_{2}(t), \\
\dot{x}_{2}(t) & =9.8 x_{1}(t)+x_{3}(t), \\
\varepsilon \dot{x}_{3}(t) & =-x_{2}(t)-x_{3}(t)+u(t)+w(t),
\end{aligned}
$$

where $\varepsilon=L_{a}$.

The equilibrium point of system (66), that is, $x_{e}=$ $\left[\begin{array}{lll}0 & 0 & 0\end{array}\right]^{T}$, corresponds to the upright rest position of the inverted pendulum. We will design a controller to balance the pendulum around its upright rest position. 
According to [36], we choose the sampling period as $T_{f}=$ $\alpha_{f} \varepsilon$, where $\alpha_{f}=0.1, \varepsilon=0.05$. Then the fast sampling discrete-time model of system (66) is in the form of (1) with

$$
\begin{aligned}
A_{11} & =\left[\begin{array}{cc}
0 & 0.1 \\
0.98 & -0.0048
\end{array}\right], \\
A_{12} & =\left[\begin{array}{c}
0 \\
0.0952
\end{array}\right], \\
A_{22} & =0.9048, \\
B_{1} & =\left[\begin{array}{c}
0 \\
0.0048
\end{array}\right], \\
B_{2} & =0.0952, \\
E_{1} & =\left[\begin{array}{c}
0 \\
0.0048
\end{array}\right], \\
E_{2} & =0.0952, \\
C_{1} & =\left[\begin{array}{ll}
1 & 1
\end{array}\right], \\
C_{2} & =1 .
\end{aligned}
$$

Solving the LMIs of Theorem 6 with $\varepsilon_{0}=0.05, \alpha^{*}=0.2$, and $\eta=1$, we have

$$
\begin{aligned}
& P_{11}=\left[\begin{array}{cc}
23.4260 & -52.6506 \\
-52.6506 & 157.2814
\end{array}\right], \\
& P_{12}=\left[\begin{array}{c}
-92.2040 \\
68.0912
\end{array}\right], \\
& P_{22}=92.9218, \\
& Z_{1}=\left[\begin{array}{ll}
-152.3819 & 187.0950
\end{array}\right], \\
& Z_{2}=-491.8613, \\
& Y_{1}=\left[\begin{array}{ll}
0.1739 & -0.1772
\end{array}\right], \\
& Y_{2}=-0.1620 .
\end{aligned}
$$

Choosing $\varepsilon=0.05 \in\left(0, \varepsilon_{0}\right]$, then we have

$$
\begin{aligned}
P(\varepsilon) & =\left[\begin{array}{ccc}
1.1713 & -2.6325 & -4.6102 \\
-2.6325 & 7.8641 & 3.4046 \\
-4.6102 & 3.4046 & 92.9218
\end{array}\right], \\
Z_{\varepsilon} & =\left[\begin{array}{lll}
-7.6191 & 9.3548 & -491.8613
\end{array}\right], \\
Y_{\varepsilon} & =\left[\begin{array}{lll}
0.0087 & -0.0089 & -0.1620
\end{array}\right] .
\end{aligned}
$$

Then we can calculate the state feedback controller gain:

$$
K_{\varepsilon}=\left[\begin{array}{lll}
-147.2017 & -43.3206 & -11.0093
\end{array}\right] .
$$

Solving the optimization problem (48) with $\varepsilon_{0}=0.05$, then we get

$$
\begin{aligned}
P_{11} & =\left[\begin{array}{cc}
0.0097 & -0.0277 \\
-0.0277 & 0.1043
\end{array}\right], \\
P_{12} & =\left[\begin{array}{ll}
-0.0122 \\
-0.0551
\end{array}\right], \\
P_{22} & =0.0710, \\
Z_{1} & =\left[\begin{array}{ll}
-0.0748 & 0.0910
\end{array}\right], \\
Z_{2} & =-0.0492, \\
Y_{1} & =\left[\begin{array}{ll}
-0.0442 & -0.0299
\end{array}\right], \\
Y_{2} & =0.0133 .
\end{aligned}
$$

Choosing $\varepsilon=0.05 \in\left(0, \varepsilon_{0}\right]$, we have

$$
\begin{aligned}
P(\varepsilon) & =\left[\begin{array}{ccc}
0.0005 & -0.0014 & -0.0006 \\
-0.0014 & 0.0052 & -0.0028 \\
-0.0006 & -0.0028 & 0.0710
\end{array}\right], \\
Z_{\varepsilon} & =\left[\begin{array}{lll}
-0.0037 & 0.0045 & -0.0492
\end{array}\right], \\
Y_{\varepsilon} & =\left[\begin{array}{lll}
-0.0022 & -0.0015 & 0.0133
\end{array}\right], \\
K_{\varepsilon} & =\left[\begin{array}{lll}
-37.7656 & -9.9051 & -1.4045
\end{array}\right],
\end{aligned}
$$

and $\mu=0.0641$, which means the capacity of disturbance tolerance of the system is $\alpha^{*}=15.5997$.

To improve the ability of disturbance rejection, solving the optimization problem (64) with $\varepsilon_{0}=0.05, \alpha^{*}=15$, we have

$$
\begin{aligned}
P_{11} & =\left[\begin{array}{cc}
0.0045 & -0.0133 \\
-0.0133 & 0.0522
\end{array}\right], \\
P_{12} & =\left[\begin{array}{c}
-0.0338 \\
0.0513
\end{array}\right], \\
P_{22} & =0.0888 \\
Z_{1} & =\left[\begin{array}{ll}
-0.0479 & 0.0363
\end{array}\right], \\
Z_{2} & =-0.6921, \\
Y_{1} & =[-0.0238-0.0405], \\
Y_{2} & =-0.0004 .
\end{aligned}
$$

Choosing $\varepsilon=0.05 \in\left(0, \varepsilon_{0}\right]$, we have

$$
P(\varepsilon)=\left[\begin{array}{ccc}
0.0002 & -0.0007 & -0.0017 \\
-0.0007 & 0.0026 & 0.0026 \\
-0.0017 & 0.0026 & 0.0888
\end{array}\right],
$$




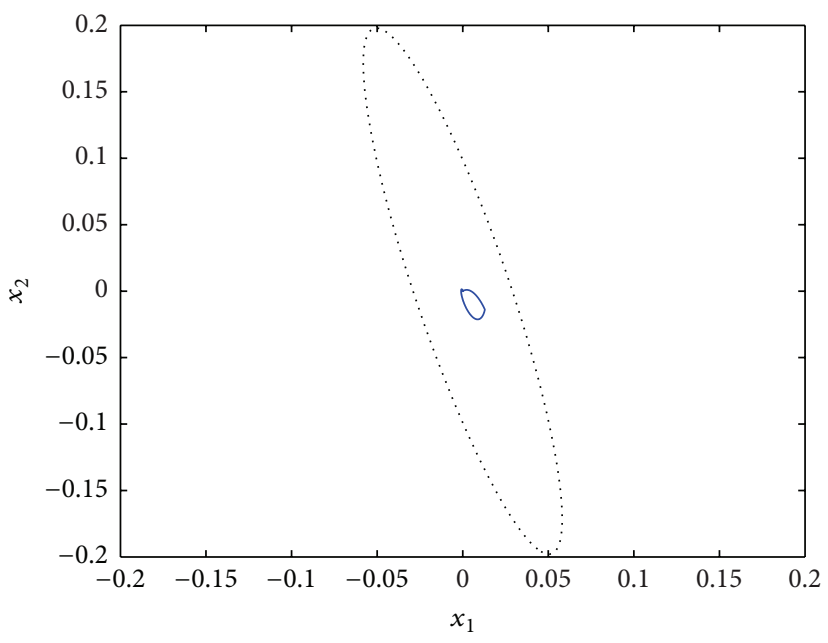

(a) The projection of trajectory to $x_{1} x_{2}$

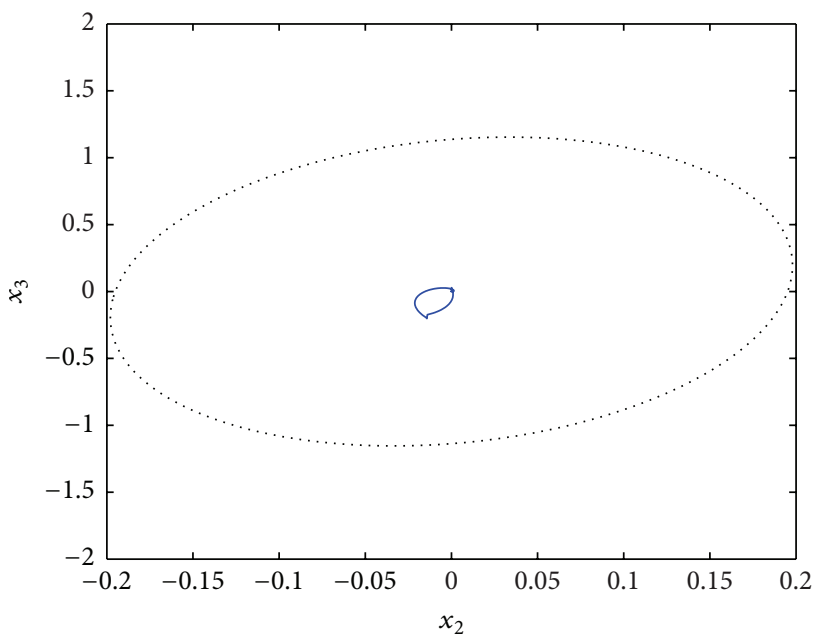

(c) The projection of trajectory to $x_{2} x_{3}$

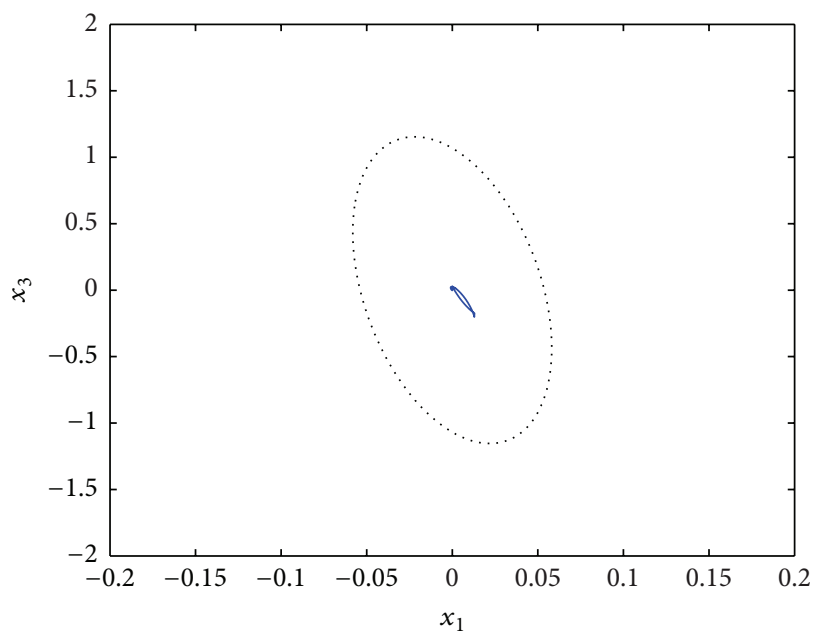

(b) The projection of trajectory to $x_{1} x_{3}$

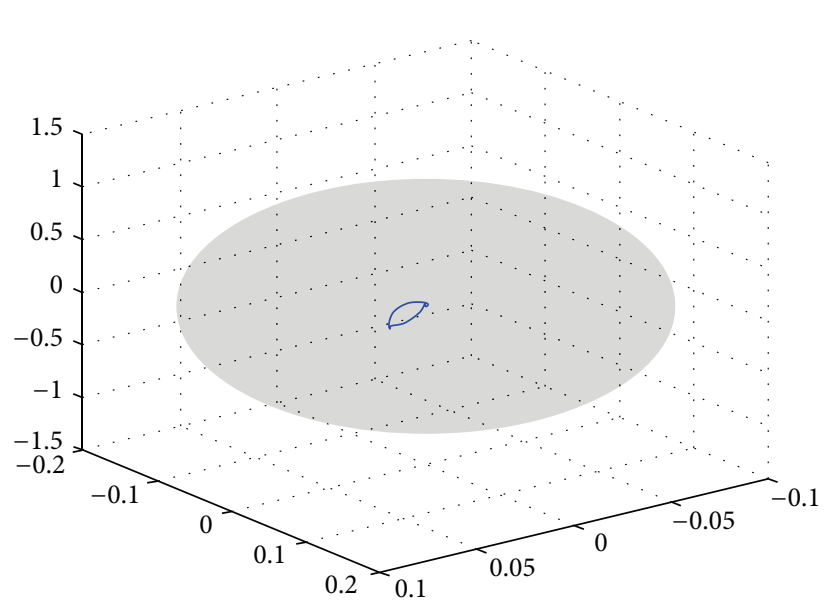

(d) The trajectory starting from the origin

FIGURE 1: The ellipsoid $\Omega\left(P^{-1}(\varepsilon), \alpha\right)$ and the trajectory starting from the origin.

$$
\begin{aligned}
Z_{\varepsilon} & =\left[\begin{array}{lll}
-0.0024 & 0.0018 & -0.6921
\end{array}\right] \\
Y_{\varepsilon} & =\left[\begin{array}{lll}
-0.0012 & -0.0020 & -0.0004
\end{array}\right], \\
K_{\varepsilon} & =\left[\begin{array}{lll}
-234.9799 & -48.4215 & -10.8701
\end{array}\right],
\end{aligned}
$$

and $\gamma=1.0369$. Thus the $L_{2}$ gain from $w$ to $z$ of the closedloop system with $x(0)=0$ is less than 1.0369.

Define a piecewise function as follows:

$$
v(k)= \begin{cases}0.3 & \text { if } 1 \leq k \leq 100 \\ 0 & \text { if } k>100\end{cases}
$$

Given $\alpha^{*}=15<15.5997$ and $\varepsilon_{0}=0.05$, it is easy to see that the disturbance $w(k)=v(k) \in W_{\alpha^{*}}^{2}$. As shown in Figure 1, under controller (4), with

$$
K_{\varepsilon}=\left[\begin{array}{lll}
-234.9799 & -48.4215 & -10.8701
\end{array}\right]
$$

the trajectory of the closed-loop system with the disturbance $\omega(k)=v(k)$ starting from the origin is bounded and converges to the origin when the disturbance disappears.

\section{Conclusion}

This paper investigated the problem of fast sampling control for singularly perturbed systems subject to actuator saturation and $L_{2}$ disturbance. A state feedback controller method was proposed such that all the trajectories of the closed-loop system starting from a bounded set will remain bounded for any singular perturbation parameter less than or equal to a predefined upper bound. Convex optimization problems were formulated to optimize the ability of disturbance tolerance and disturbance rejection, respectively. The presented example has illustrated the significance and validity of the proposed approaches. 


\section{Conflict of Interests}

The authors declare that there is no conflict of interests regarding the publication of this paper.

\section{Acknowledgments}

This work was supported by the National Natural Science Foundation of China $(61374043,61303183)$, the Jiangsu Provincial Natural Science Foundation of China (BK20130204, BK20130205), the China Postdoctoral Science Foundation Funded Project (2013M530278, 2014T70558), and the Fundamental Research Funds for the Central Universities (2013QNA50, 2013RC10, and 2013RC12).

\section{References}

[1] P. V. Kokotovic, H. K. Khalil, and J. O’Reilly, Singular Perturbation Methods in Control: Analysis and Design, Academic, New York, NY, USA, 1986.

[2] R. Ramírez-Betancour, C. R. Fuerte-Esquivel, and T. Van Cutsem, "A two-time scale simulation for dynamic analysis of power systems," Electric Power Systems Research, vol. 83, no. 1, pp. 185-195, 2012.

[3] M. Heidarinejad, J. Liu, D. M. de la Peña, J. F. Davis, and P. D. Christofides, "Multirate Lyapunov-based distributed model predictive control of nonlinear uncertain systems," Journal of Process Control, vol. 21, no. 9, pp. 1231-1242, 2011.

[4] X. Chen, M. Heidarinejad, J. Liu, D. M. de la Peña, and P. D. Christofides, "Model predictive control of nonlinear singularly perturbed systems: application to a large-scale process network," Journal of Process Control, vol. 21, no. 9, pp. 1296-1305, 2011.

[5] K. A. Rao and D. S. Naidu, "Singular perturbation method applied to the open-loop discrete optimial control problem," Optimal Control Applications and Methods, vol. 3, no. 2, pp. 121131, 1982.

[6] H. Kando and T. Iwazumi, "Sub-optimal control of discrete regulation problems via time-scale decomposition," International Journal of Control, vol. 37, no. 6, pp. 1323-1347, 1983.

[7] J. Dong and G.-H. Yang, "Robust $H_{\infty}$ control for standard discrete-time singularly perturbed systems," IET Control Theory \& Applications, vol. 1, no. 4, pp. 1141-1148, 2007.

[8] G. Wang, Q. Zhang, and V. Sreeram, " $H_{\infty}$ control for discretetime singularly perturbed systems with two Markov processes," Journal of the Franklin Institute, vol. 347, no. 5, pp. 836-847, 2010.

[9] G. Blankenship, "Singularly perturbed defference equations in optimal control problems," IEEE Transactions on Automatic Control, vol. 26, no. 4, pp. 911-917, 1981.

[10] B. Litkouhi and H. Khalil, "Infinite-time regulations for singularly perturbed defference equations," International Journal of Control, vol. 39, no. 3, pp. 587-598, 1984.

[11] J. Dong and G.-H. Yang, " $H_{\infty}$ control for fast sampling discretetime singularly perturbed systems," Automatica, vol. 44, no. 5, pp. 1385-1393, 2008.

[12] S. Xu and G. Feng, "New results on $H_{\infty}$ control of discrete singularly perturbed systems," Automatica, vol. 45, no. 10, pp. 2339-2343, 2009.

[13] T. Hu and Z. Lin, Control Systems with Actuator Saturation: Analysis and Design, Birkhäuser, Boston, Mass, USA, 2001.
[14] Z. Zuo, D. W. C. Ho, and Y. Wang, "Fault tolerant control for singular systems with actuator saturation and nonlinear perturbation,” Automatica, vol. 46, no. 3, pp. 569-576, 2010.

[15] Q. Wang, B. Zhou, C. Wen, and G.-R. Duan, "Output feedback gain scheduled control of actuator saturated linear systems with applications to the spacecraft rendezvous," Journal of the Franklin Institute, vol. 351, no. 11, pp. 5015-5033, 2014.

[16] B. Zhou, Q. Wang, Z. Lin, and G.-R. Duan, "Gain scheduled control of linear systems subject to actuator saturation with application to spacecraft rendezvous," IEEE Transactions on Control Systems Technology, vol. 22, no. 5, pp. 2031-2038, 2014.

[17] B. Zhou, H. Gao, Z. Lin, and G.-R. Duan, "Stabilization of linear systems with distributed input delay and input saturation," Automatica, vol. 48, no. 5, pp. 712-714, 2012.

[18] T. Hu, Z. Lin, and B. M. Chen, "An analysis and design method for linear systems subject to actuator saturation and disturbance," Automatica, vol. 38, no. 2, pp. 351-359, 2002.

[19] Y.-Y. Cao, Z. Lin, and D. G. Ward, "An antiwindup approach to enlarging domain of attraction for linear systems subject to actuator saturation," IEEE Transactions on Automatic Control, vol. 47, no. 1, pp. 140-145, 2002.

[20] P.-L. Liu, "Stabilization of singularly perturbed multiple-timedelay systems with a saturating actuator," International Journal of Systems Science, vol. 32, no. 8, pp. 1041-1045, 2001.

[21] G. Garcia and S. Tarbouriech, "Control of singularly perturbed systems by bounded control," in Proceedings of the American Control Conference, vol. 5, pp. 4482-4487, IEEE, Denver, Colo, USA, June 2003.

[22] H.-H. Xin, D. Wu, D.-Q. Gan, and J.-J. Qiu, "A method for estimating the stability region of singular perturbation systems with saturation nonlinearities," Acta Automatica Sinica, vol. 34, no. 12, pp. 1549-1555, 2008.

[23] H. Xin, D. Gan, M. Huang, and K. Wang, "Estimating the stability region of singular perturbation power systems with saturation nonlinearities: an linear matrix inequality-based method," IET Control Theory \& Applications, vol. 4, no. 3, pp. 351-361, 2010.

[24] I. Lizarraga, S. Tarbouriech, and G. Garcia, "Control of singularly perturbed systems under actuator saturation," in Proceedings of the 16th Triennial World Congress of International Federation of Automatic Control (IFAC '05), pp. 243-248, Prague, Czech Republic, July 2005.

[25] W. Feng, "Characterization and computation for the bound $\varepsilon^{*}$ in linear time-invariant singularly perturbed systems," Systems \& Control Letters, vol. 11, no. 3, pp. 195-202, 1988.

[26] S. Sen and K. B. Datta, "Stability bounds of singularity perturbed systems," IEEE Transactions on Automatic Control, vol. 38, no. 2, pp. 302-304, 1993.

[27] L. Cao and H. M. Schwartz, "Complementary results on the stability bounds of singularly perturbed systems," IEEE Transactions on Automatic Control, vol. 49, no. 11, pp. 2017-2021, 2004.

[28] T.-H. S. Li and J.-H. Li, "Stabilization bound of discrete twotime-scale systems," Systems \& Control Letters, vol. 18, no. 6, pp. 479-489, 1992.

[29] W. Q. Liu, M. Paskota, V. Sreeram, and K. L. Teo, "Improvement on stability bounds for singularly perturbed systems via state feedback," International Journal of Systems Science, vol. 28, no. 6, pp. 571-578, 1997.

[30] J.-S. Chiou, F.-C. Kung, and T.-H. S. Li, "An infinite $\varepsilon$-bound stabilization design for a class of singularly perturbed systems," IEEE Transactions on Circuits and Systems I: Fundamental Theory and Applications, vol. 46, no. 12, pp. 1507-1510, 1999. 
[31] G.-H. Yang and J. X. Dong, "Control synthesis of singularly perturbed fuzzy systems," IEEE Transactions on Fuzzy Systems, vol. 16, no. 3, pp. 615-629, 2008.

[32] C. Yang and Q. Zhang, "Multiobjective control for T-S fuzzy singularly perturbed systems," IEEE Transactions on Fuzzy Systems, vol. 17, no. 1, pp. 104-115, 2009.

[33] C. Y. Yang, J. Sun, and X. P. Ma, "Stabilization bound of singularly perturbed systems subject to actuator saturation," Automatica, vol. 49, no. 2, pp. 457-462, 2013.

[34] H. Hindi and S. Boyd, "Analysis of linear systems with saturating using convex optimization," in Preceedings of the 37th IEEE Conference on Decision and Control, pp. 903-908, Tampa, Fla, USA, 1998.

[35] S. H. Zak and C. A. Maccarley, "State-feedback control of nonlinear systems," International Journal of Control, vol. 43, no. 5, pp. 1497-1514, 1986.

[36] H. Kando and T. Iwazumi, "Multirate digital control design of an optimal regulartor via singular perturbation theory," International Journal of Control, vol. 44, no. 6, pp. 1555-1578, 1986. 


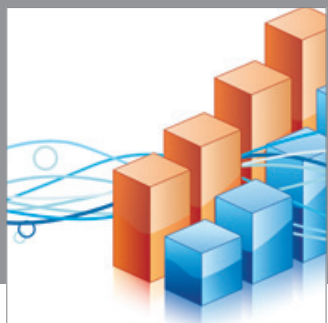

Advances in

Operations Research

mansans

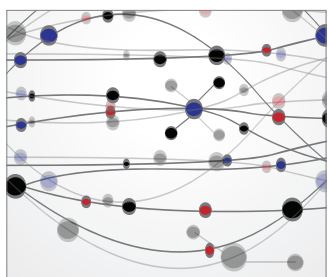

The Scientific World Journal
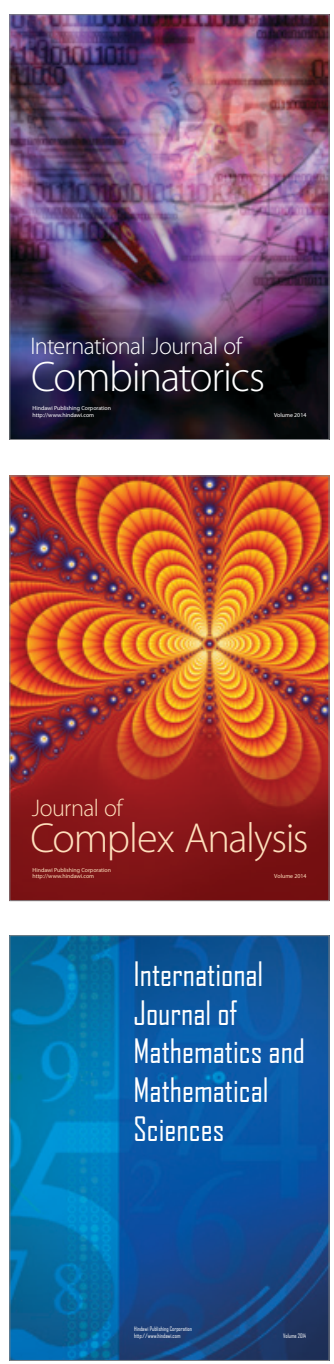
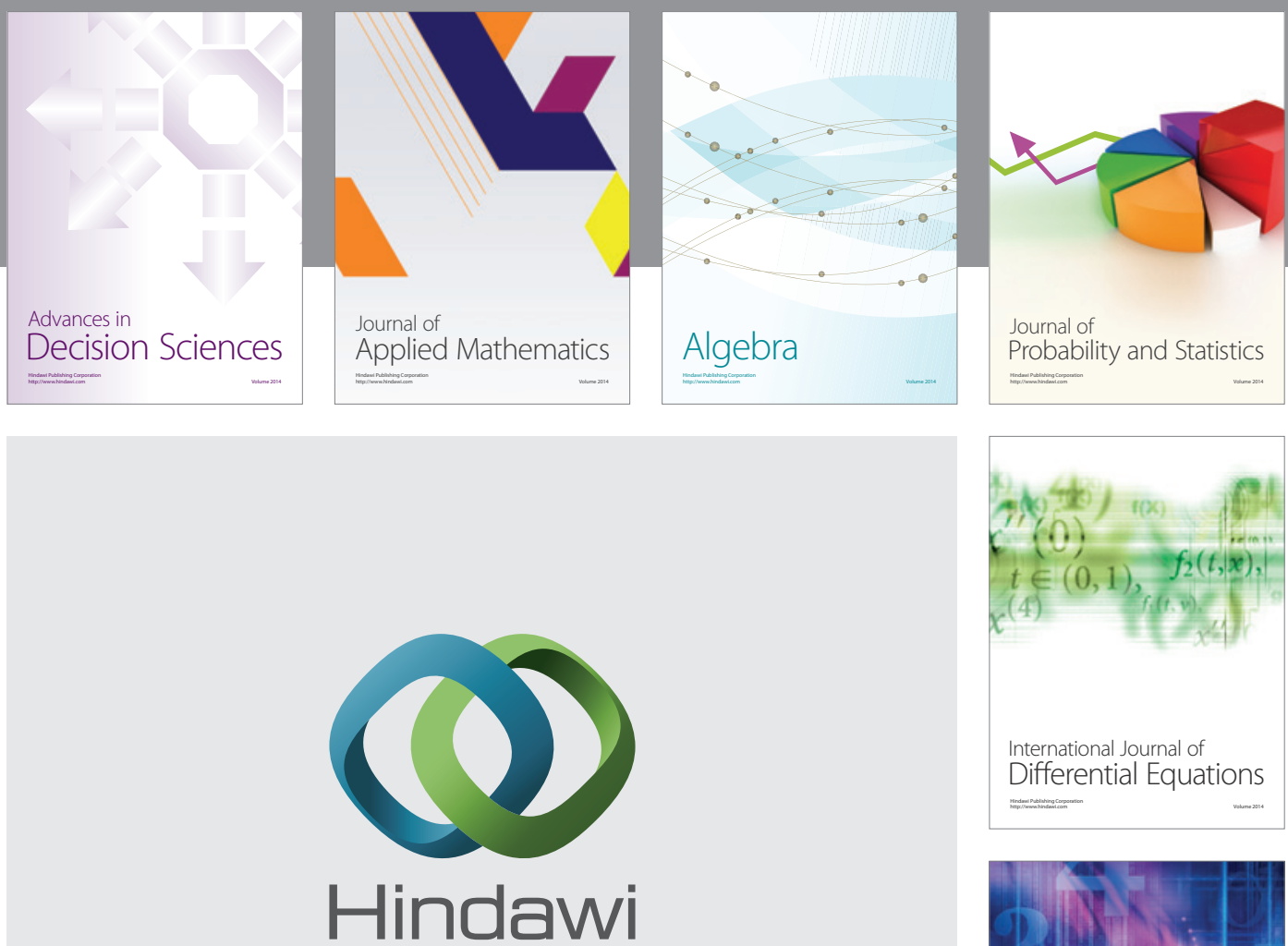

Submit your manuscripts at http://www.hindawi.com
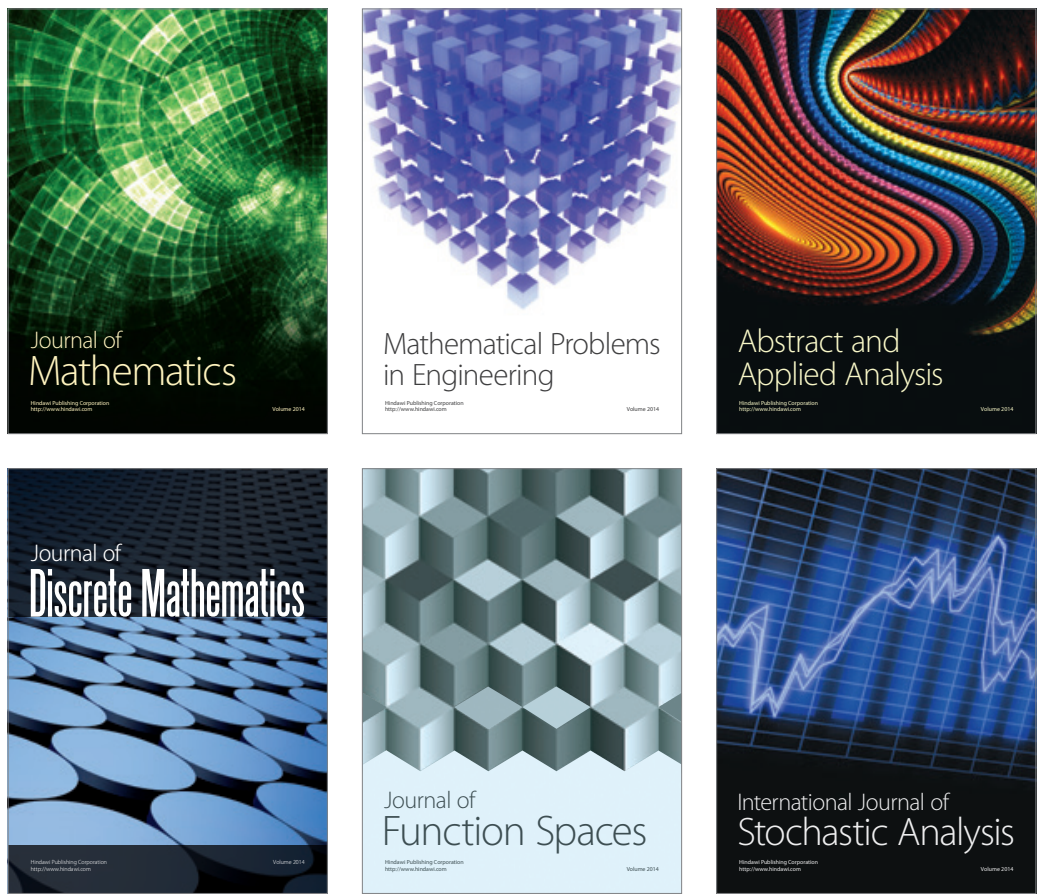

Journal of

Function Spaces

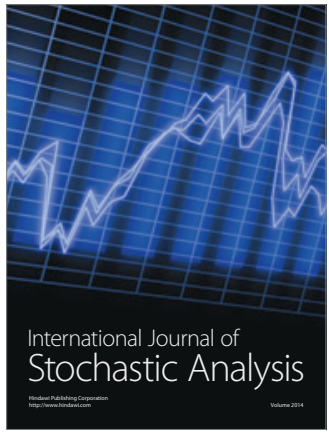

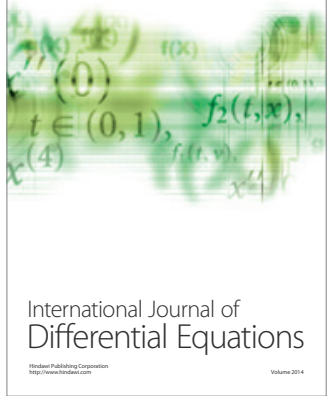
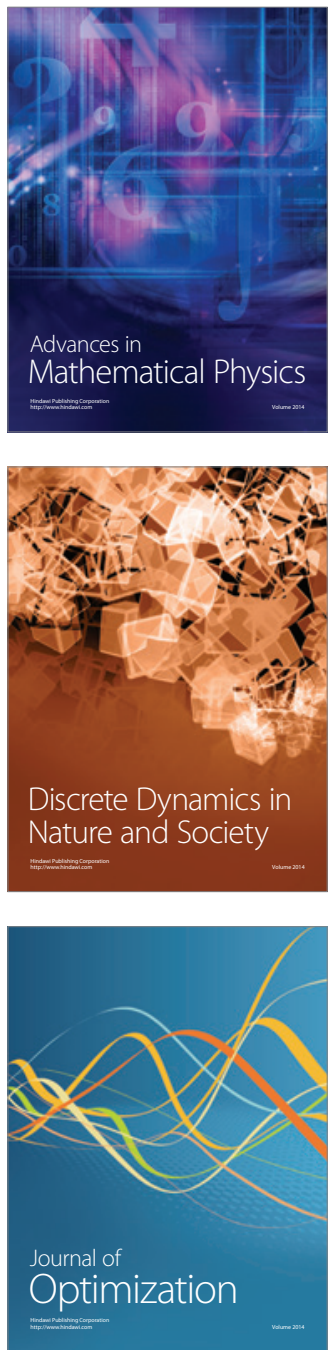